\title{
A rare case of juvenile amyotrophic lateral sclerosis
}

\author{
Muhittin Bodur ${ }^{1 \oplus}$, Rabia Tütüncü Toker ${ }^{1 \oplus}$, Ayşe Nazlı Başak ${ }^{2 \oplus}$, Mehmet Sait Okan ${ }^{1 \oplus}$ \\ ${ }^{1}$ Division of Pediatric Neurology, Department of Pediatrics, Uludag University Faculty of Medicine, Bursa; ${ }^{2}$ Suna and Inan Kıraç \\ Foundation, Neurodegeneration Research Laboratory, School of Medicine, Koç University Translational Medicine Research Center, \\ Istanbul, Turkey.
}

\begin{abstract}
Background. Amyotrophic lateral sclerosis (ALS) is a chronic motor neuron disease characterised by progressive weakness in striated muscles resulting from the destruction of neuronal cells. The term juvenile ALS (JALS) is used for patients whose symptoms start before 25 years of age. JALS may be sporadic or familial.
\end{abstract}

Case. Here, we present a sporadic case of JALS because of its rarity in children. The heterozygous p.Pro525Leu (c.1574C > T) variation was identified in the fused in sarcoma (FUS) gene.

Conclusion. The p.Pro525Leu mutation in the FUS gene has been detected in patients with ALS, characterised by early onset and a severely progressive course.

Key words: juvenile amyotrophic lateral sclerosis, FUS gene.

Amyotrophic lateral sclerosis (ALS) is a chronic motor neuron disease characterised by progressive weakness in striated muscles resulting from the destruction of neuronal cells. ${ }^{1}$ It is a progressive disease characterised by the co-existence of upper and lower motor neuron findings. ${ }^{1}$ The term juvenile ALS (JALS) is used for patients whose symptoms start before they reach 25 years of age. ${ }^{1}$ These cases usually have prolonged survival. JALS may be sporadic or familial. ${ }^{1}$ Here, we present a sporadic case of JALS because of its rarity in children.

\section{Case Report}

A 17-year-old boy was admitted to our clinic with complaints of weakness and difficulty in speaking and swallowing. Weakness in the right foot had started approximately six months previously and had spread to the right

\footnotetext{
Muhittin Bodur

dr_muhittin@hotmail.com
}

Received 13th November 2019,

revised 22nd January 2020, 5th April 2020,

19th April 2020, 2nd June 2020, 15th July 2020,

12th August 2020, 7th September 2020,

14th September 2020, accepted 15th October 2020. arm, left arm and left leg in the three weeks before the admission. Before these complaints, he had no previous history of serious illness, muscle pain, trauma or adverse reaction to vaccinations, and he had no recent infections. His medical history revealed that he was born at term with a birthweight of $2,000 \mathrm{~g}$, and his neuromotor development was normal. There has been no consanguineous marriage in the family history, nor any disease similar to ALS. On physical and neurological examination, he had mild dysarthric speech and tongue fasciculation. His uvula was slightly deviated to the left, and his retching reflex was decreased. Muscle strength of right upper limb was 3/5 and left upper limb was 4/5 (in deltoids, biceps, triceps and hands). Muscle strength of right lower limb was $2 / 5$ in the proximal muscles (in iliopsoas, hamstring, quadriceps femoris) and $1 / 5$ in the distal muscles (in tibialis anterior, gastrocnemius) and left lower limb was $4 / 5$. His tendon reflexes were hypoactive in the left lower and upper limbs and hyperactive in the right lower and upper limbs. His sensory examination was normal. There were atrophy findings in the right-upper and lower limbs. Laboratory findings established the following: hemogram and peripheral smear were normal; 
creatine kinase 179 (40-220 IU/L); sedimentation $6 \mathrm{~mm} / \mathrm{h}$; blood amino acids, tandem MS/MS and lactate were normal; vasculitis screening was found to be normal; and HIV serology was negative. Electromyography (EMG) showed a reduced amplitude of the motor potentials in the examined motor nerves. Fibrillation potential, sharp waves and prolonged polyphasic motor unit potentials (MUPs) were seen in bilateral tibialis anterior, bilateral gastrocnemius, right biceps, right deltoid, genioglossus and rectus abdominis muscles. The findings were evaluated as diffuse anterior horn involvement. Cranial MRI (Fig. 1) showed increased linear signal intensity on T2W images along both internal capsule hind legs to mesencephalon; the diffusion-based examination showed slight diffusion restriction in this area, and the change in intensity and diffusion restriction observed in the bilateral corticospinal tract was evaluated in terms of ALS with clinical findings. Cervical, thoracic and lumbar MRI were normal. Before genetic testing, the patient was diagnosed with JALS based on the clinical, laboratory, electrophysiological and MRI findings. The family was informed about ALS, treatment (riluzole) was commenced, and genetic examination was performed. Four months after diagnosis, the patient became bedridden. He received a tracheostomy and required mechanical ventilation. Eleven months after diagnosis, he died as a result of pneumonia and sepsis. The heterozygous p.Pro525Leu (c.1574C $>$ T) mutation was identified in the fused in sarcoma (FUS) gene. Whole exome sequencing (WES) was confirmed by Sanger sequencing, and segregation analysis in the patient's parents showed that this variant was de novo. The parents were not carriers of the mutation. Informed consent was received from the family.

\section{Discussion}

ALS is a neurodegenerative disease, which usually starts in adulthood and is characterised

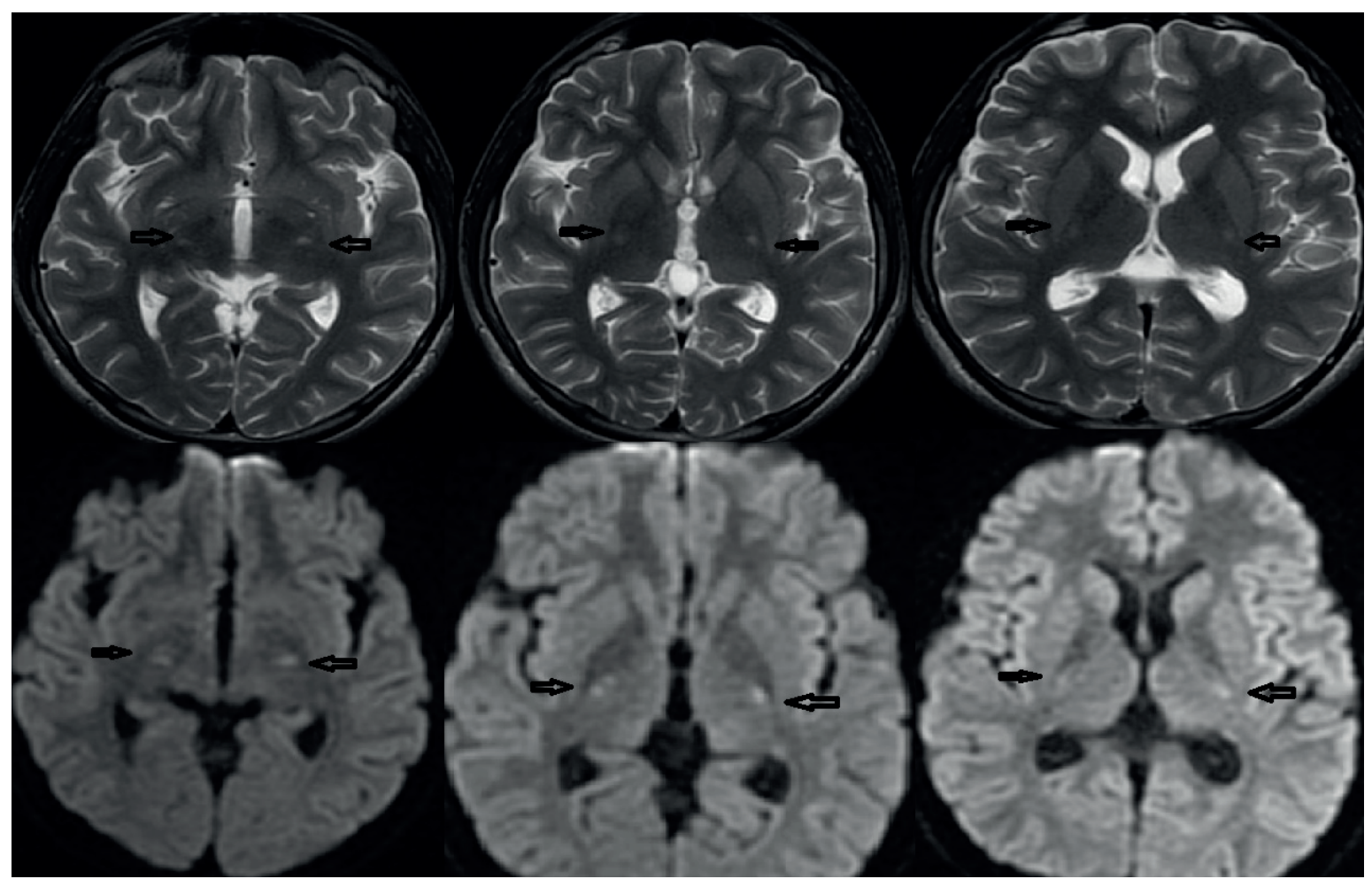

Fig. 1. MRI images of the case: increased linear signal intensity on T2W images along both internal capsule hind legs to the mesencephalon; the diffusion-based examination: slight diffusion restriction in this area. 
by progressive muscle weakness and muscle atrophy. ${ }^{2}$ Approximately $10 \%$ of ALS cases are familial, and $90 \%$ are sporadic, but the majority of JALS cases have a genetic origin. ${ }^{3}$ ALS is characterised by progressive damage to motor neurons responsible for the control of striated muscle groups in the cortex, brain stem and spinal cord. ALS progresses with loss of upper and lower motor neurons. Progressive muscle atrophy and diffuse paralysis develop over time. Death from ALS is due to respiratory failure $^{4}$ and occurs within an average of 3-5 years from the onset of the disease. Although the genetic background of the disease is well understood in familial cases, the underlying pathology is still not clear, and there is no effective treatment. ${ }^{5}$ ALS is diagnosed clinically. Muscular atrophy and vigorous deep tendon reflexes with focal-onset progressive course and weakness spreading to other extremities over time suggest ALS as the underlying cause. ${ }^{6}$ Pyramidal pathway involvement may be seen in ischemic events, demyelinating disorders or infections. However, the change in intensity observed in our patient's bilateral corticospinal tract on cranial MRI has been reported among the characteristic clinical findings in the diagnosis of ALS. The World Federation of Neurology identified El Escorial criteria (1994) for use in the diagnosis of $\mathrm{ALS}^{7}$ and these were revised in $2000 .{ }^{8}$ These criteria divide the motor system into 4 anatomic regions: bulbar, cervical, thoracic, and lumbosacral. Clinical evidence of upper motor neuron (UMN) and lower motor neuron (LMN) pathology is sought within each region; the certainty of diagnosis depends on how many regions reveal UMN and/or LMN pathology. ${ }^{9}$ Patients with signs of LMN degeneration (weakness, atrophy and clinical fasciculations) and UMN degeneration (spasticity, pathologic reflexes, etc.) may be suspected as having ALS. Careful history, physical and neurological examination must search for further clinical evidence of LMN and UMN signs in four regions of the central nervous system. ${ }^{7}$ According to the El Escorial criteria, upper and lower motor neuron degeneration findings should be present at the same site for ALS diagnosis. The clinical picture should progressively spread from one side to the other. Other factors that may explain lower or upper motor neuron findings should be excluded in the light of laboratory, electrophysiological or neuroimaging data. ${ }^{7}$ In the examination of our patient, there were bulbar involvement (dysarthric speech, fasciculation in the tongue, difficulty in swallowing) and weakness that started from the lower right limb and spread to other limbs. In addition, there were findings of lower motor neuron involvement (weakness, muscle atrophy, DTR hypoactivity) in the left lower limb (lumbosacral) and left upper limb (cervical), and findings related to the involvement of upper motor neurons (weakness, DTR hyperactivity) in the right lower limb (lumbosacral) and right upper limb (cervical) were detected. Electromyography (EMG) is the most important component of the electrodiagnostic evaluation in ALS. To support a diagnosis of ALS, the needle electrode examination should reveal decreased motor unit recruitment with rapid firing of a reduced number of motor units, and/or large amplitude, long duration MUP with or without evidence of remodelling (increased number of phases) in combination with abnormal spontaneous activity including positive sharp waves (PSWs), fibrillations, and/or fasciculation potentials (FP). ${ }^{9}$ In this case, a reduced amplitude was observed in the examined motor nerves; intense denervation findings (fibrillation potentials and sharp waves) were obtained in the extremity muscles (cervical, lumbosacral) and midline muscles tract (bulbar, thoracic); and prolonged polyphasic motor unit potentials (MUPs) were observed. These findings were consistent with the EMG findings of ALS, evidence of chronic neurogenic change and evidence of acute denervation. In this case according to the examination and EMG findings, the findings of lower motor neuron in the bulbar and thoracic region, and both upper and lower motor neuron in the cervical and lumbosacral region were detected. As a result of these findings, this case was evaluated as clinical probable ALS. In this case, clinical, laboratory, electrophysiological 
and imaging findings were consistent with the El Escorial criteria, and no other cause of upper and lower motor neuron destruction could be detected. To date, there are 29 genes that are considered to be causes of or highly correlated with ALS. ${ }^{10}$ FUS mutations have been observed in patients with JALS, where symptoms start before the age of 25 years. ${ }^{11}$ The FUS gene is located on chromosome 16p11.2 and consists of 14 introns and 15 exons. It encodes the FUS protein of 526 amino acid residues that belong to the multifunctional DNA/RNA-binding proteins. The FUS protein is ubiquitously expressed in the cell nucleus and cytoplasm and continuously moves between the two areas. It is involved in cell proliferation, DNA repair, transcription regulation, RNA transport and microRNA processing. ${ }^{12}$ FUS mutations are reported to be responsible for $4 \%$ of familial $\mathrm{ALS}^{13}$ and less than $1 \%$ of sporadic ALS cases, ${ }^{14}$ which illustrates their rarity. Among these mutations, p.P525L FUS mutation has been consistently associated with young-onset, rapid disease course and high proportions of de novo mutations in sporadic cases. ${ }^{15}$ In this case, the heterozygous p.Pro525Leu (c.1574C $>$ T) mutation was identified in the FUS gene, and analysis in the patient's parents showed that this variant was de novo. In reviewing the literature, 6 cases with P525L mutation were observed in pediatric ALS cases (Table I). In accordance with the literature our case showed early onset and rapid progression, and the patient died 11 months after diagnosis. There is no effective treatment for the disease. Riluzole, which has been used since 1996, is a glutamate antagonist and is the only drug that prolongs survival. A daily dose of $100 \mathrm{mg}$ Riluzole prolongs survival in ALS patients by an average of 3-5 months. ${ }^{16}$ There is a need for new treatment methods that will increase the survival rate for patients with the disease. Although JALS is rare in children, it should be kept in mind, especially in cases with upper motor and lower motor neuron findings.

\section{Author contribution}

The authors confirm contribution to the paper as follows: study conception and design: Muhittin Bodur, Rabia Tütüncü Toker, Ayşe Nazlı Başak , Mehmet Sait Okan; data collection: Muhittin Bodur, Rabia Tütüncü Toker, Ayşe Nazlı Başak, Mehmet Sait Okan; analysis and interpretation of results: Muhittin Bodur, Rabia Tütüncü Toker, Ayşe Nazlı Başak, Mehmet Sait Okan; draft manuscript preparation: Muhittin Bodur, Rabia Tütüncü Toker, Ayşe Nazlı Başak, Mehmet Sait Okan

All authors reviewed the results and approved the final version of the manuscript.

\section{Conflicts of interest}

The authors declare no conflict of interest.

\section{REFERENCES}

1. Aggarwal A, Shashiraj. Juvenile amyotrophic lateral sclerosis. Indian J Pediatr 2006; 73: 225-226.

2. Rowland LP, Shneider NA. Amyotrophic lateral sclerosis. N Engl J Med 2001; 344: 1688-1700.

Table I. P525L FUS mutations in pediatric ALS cases.

\begin{tabular}{lccccc}
\hline Age at onset, year & Gender & Sporadic/Famialial & Site of onset & Disease duration (months) & Reference \\
\hline 11 & F & S & Limb & 14 & 16 \\
13 & F & S & Limb & 20 & 17 \\
13 & M & N/A & Limb & 23 & 18 \\
16 & M & F & Bulbar & 12 & 19 \\
17 & F & S & Bulbar & 24 & 20 \\
17 & M & S & Limb & 15 & 20 \\
17 & M & S & Limb and Bulbar & 11 & This report
\end{tabular}

F: female, M: male, S: sporadic, F: famialial, N/A: not available. 
3. Emery AEH, Holloway S. Familial motor neuron disease. In: Rowland LP (ed). Human Motor Neuron Disease. (Advances in Neurology). New York: Raven Press, 1982; 36: 139-147.

4. Rizzo F, Riboldi G, Salani S, et al. Cellular therapy to target neuroinflammation in amyotrophic lateral sclerosis. Cell Mol Life Sci 2014; 71: 999-1015.

5. Thomsen GM, Gowing G, Svendsen S, Svendsen $\mathrm{CN}$. The past, present and future of stem cell clinical trials for ALS. Exp Neurol 2014; 262(Pt B): 127-137.

6. Chio A. Risk factors in the early diagnosis of ALS: European epidemiological studies. Amyotroph Lateral Scler Other Motor Neuron Disord 2000; 1(Suppl 1): 13-18.

7. Brooks BR. El Escorial World Federation of Neurology criteria for the diagnosis of amyotrophic lateral sclerosis. Subcommittee on Motor Neuron Diseases/Amyotrophic Lateral Sclerosis of the World Federation of Neurology Research Group on Neuromuscular Diseases and the El Escorial "Clinical limits of amyotrophic lateral sclerosis" workshop contributors. J Neurol Sci 1994; 124(Suppl): 96-107.

8. Brooks BR, Miller RG, Swash M, Munsat TL; World Federation of Neurology Research Group on Motor Neuron Diseases. El Escorial revisited: revised criteria for the diagnosis of amyotrophic lateral sclerosis. Amyotroph Lateral Scler Other Motor Neuron Disord 2000; 1: 293-299.

9. Joyce NC, Carter GT. Electrodiagnosis in persons with amyotrophic lateral sclerosis. PM R 2013; 5( Suppl 5): S89-S95.

10. Chia R, Chiò A, Traynor BJ. Novel genes associated with amyotrophic lateral sclerosis: diagnostic and clinical implications. Lancet Neurol 2018; 17: 94-102.

11. Liu ZJ, Lin HX, Liu GL, et al. The investigation of genetic and clinical features in Chinese patients with juvenile amyotrophic lateral sclerosis. Clin Genet 2017; 92: 267-273.
12. Mackenzie IR, Rademakers R, Neumann M. TDP43 and FUS in amyotrophic lateral sclerosis and frontotemporal dementia. Lancet Neurol 2010; 9: 995-1007.

13. Kwiatkowski TJ Jr, Bosco DA, Leclerc AL, et al. Mutation in the FUS/TLS gene on chromosome 16 cause familial amyotrophic lateral sclerosis. Science 2009; 323: 1205-1208

14. Lai SL, Abramzon Y, Schymick JC, et al. FUS mutations in sporadic amyotrophic lateral sclerosis. Neurobiol Aging 2011; 32: 550.e1-550.e4.

15. Conte A, Lattante S, Zollino M, et al. P525L FUS mutation is consistently associated with a severe form of juvenile amyotrophic lateral sclerosis. Neuromuscul Disord 2012; 22: 73-75.

16. Miller RG, Mitchell JD, Lyon M, Moore DH. Riluzole for amyotrophic lateral sclerosis (ALS) /motor neuron disease (MND). Amyotroph Lateral Scler Other Motor Neuron Disord 2003; 4: 191-206.

17. Huang EJ, Zhang J, Geser F, et al. Extensive FUSimmunoreactive pathology in juvenile amyotrophic lateral sclerosis with basophilic inclusions. Brain Pathol 2010; 20: 1069-1076.

18. Zou ZY, Liu MS, Li XG, Cui LY. Mutations in SOD1 and FUS caused juvenile-onset sporadic amyotrophic lateral sclerosis with aggressive progression. Ann Transl Med 2015; 3: 221.

19. Chio A, Restagno G, Brunetti M, et al; ITALSGEN Consortium. Two Italian kindreds with familial amyotrophic lateral sclerosis due to FUS mutation. Neurobiol Aging 2009; 30: 1272-1275.

20. Naumann M, Peikert K, Gunther R, et al. Phenotypes and malignancy risk of different FUS mutations in genetic amyotrophic lateral sclerosis. Ann Clin Transl Neurol 2019; 6: 2384-2394. 